\title{
Modelo para la evaluación de alternativas de localización de una PTAR para una ciudad en el Valle del Cauca Colombia.
}

\author{
Model to evaluate alternatives to locate a WWTP for a City in Valle del Cauca \\ Colombia. \\ Diego Leon Peña Orozco, Camilo Ordoñez Rodríguez, Cristian Camilo Arana Arias \\ Ingeniería Industrial, Universidad del Valle, Cali, Colombia \\ . Correo-e: jdiego.l.penalcorreounivalle.edu.co
}

\begin{abstract}
Resumen-Se aborda el problema de ubicación de una planta de tratamiento de aguas residuales para una ciudad en el contexto de tres alternativas de localización, con el fin de determinar la ubicación que genera un menor costo de transporte de aguas residuales desde el origen hasta cada una de las ubicaciones propuestas. Para la evaluación, se tiene en cuenta el diseño de la red actual y sus características, además de los aspectos legales que rigen las tarifas aplicadas a los servicios de acueducto y alcantarillado.
\end{abstract}

Palabras Clave-Aguas residuales, diseño, localización, red.

Abstract-There is a problem about to establish the best location for a treatment plant wastewater between three alternatives possible. The alternative locations are evaluated in order to determine which one is the one that generates a lower transport cost of wastewater from the source to the final location. This evaluation takes into account the current network design and features including the legal tariff applied from the government for these services.

Keywords-Design, location, network, wastewater.

\section{NOMENCLATURA}

PTAR: planta de tratamiento de agua residuales, CMA: costos medios de administración, CMO: costos medios de operación, CMI: costos medios de inversión, CMTAL: costos medios de tasas ambientales, CMLP: costo medio de largo plazo, DBO: demanda biológica de oxígeno, SST: cargas de sólidos suspendidos totales. RAS: reglamento técnico del sector de agua potable, DANE: departamento administrativo nacional de estadística, IPC: índices de precios al consumidor. GAMS: sistema general de modelaje algebraico, ROA: rentabilidad sobre activos, CVC: Corporación autónoma regional del Valle del Cauca.

\section{INTRODUCCIÓN}

La decisión de ubicación de una PTAR es tratada en este trabajo como un problema de red con una función objetivo de minimización de costos de transporte, el cual considera aspectos tarifarios aplicables por el gobierno y la proyección de crecimiento poblacional, que puede afectar la decisión de ubicación en términos de los flujos que deben administrarse dentro de la red. Se consideran los siguientes tres modelos:
A. Modelo de costo de transporte.
B. Modelo tarifario.
C. Modelo de proyección poblacional.

Estos modelos son empleados cada uno con distinto objetivo, por tal razón se plantea un modelo integrado, partiendo del costo de transporte del agua del punto $\mathrm{i}$ al punto $\mathrm{j}$ basado en las tarifas que rigen el costo de transportar el agua y los subsidios por estrato aplicados por el gobierno. De igual manera se pretende realizar la proyección de la densidad poblacional de la ciudad y su aporte adicional de carga a la red de distribución de agua residual. 


\section{CONTENIDO}

La programación lineal [1] constituye un importante campo de la optimización por varias razones, muchos problemas prácticos de la investigación de operaciones pueden plantearse como problemas de programación lineal. Algunos

casos especiales de programación lineal, tales como los problemas de flujo de redes y problemas de flujo de mercancías, se consideraron en el desarrollo de las matemáticas lo suficientemente importantes como para generar por si mismos mucha investigación sobre algoritmos especializados en su solución.

El problema de localización [2] tiene su principio en ubicar uno o varios servicios para cubrir la demanda de una serie de puntos conocidos, usualmente denominados puntos de demanda, optimizando alguna medida de efectividad. En la literatura se puede encontrar un gran número de trabajos dedicados al estudio de este tipo de problemas, dependiendo del planteamiento de esos problemas, los procedimientos para estimarlos se clasifican en modelos de localización continua, programación entera y ubicación de redes.

Los modelos de ubicación de red: En los modelos de ubicación de red se calcula la distancia como el camino más corto. Se establecen unos nodos los cuales representan puntos de demanda y el lugar de la planta potencial es equivalente a un subconjunto de nodos y arcos. El modelo de ubicación es muy amplio y tiene muchas aplicaciones: redes de distribución eléctrica, de comunicación, regadío etc. De entre los infinitos problemas posibles, podemos distinguir familias de problemas: Flujo a costo mínimo, flujo máximo por una red, modelo de la ruta más corta [3].

El modelo de flujo máximo: Se trata de enlazar un nodo fuente y un nodo destino a través de una red de arcos dirigidos. Cada arco tiene una capacidad máxima de flujo admisible. El objetivo es el de obtener la máxima capacidad de flujo entre la fuente y el destino.

Modelo de la ruta más corta: Este modelo busca establecer una red conexa y no dirigida con dos nodos especiales llamados origen y destino. A cada ligadura (arco no dirigido) se asocia una distancia no negativa. El objetivo es encontrar la ruta más corta (la trayectoria con la mínima distancia total) del origen al destino.

Problema del flujo de costo mínimo: El problema de flujo de costo mínimo tiene una posición medular entre los problemas de optimización de redes; primero, abarca una clase amplia de aplicaciones y segundo, su solución es muy eficiente. Igual que el problema del flujo máximo, toma en cuenta un flujo en una red con capacidades limitadas en sus arcos. Igual que el problema de la ruta más corta, considera un costo (o distancia) para el flujo a través de un arco. Igual que el problema de transporte o el de asignación, puede manejar varios orígenes (nodos-fuente) y varios destinos (nodos-demandas) para el flujo, de nuevo con costos asociados. De hecho, estos cuatro problemas son casos especiales del problema de flujo de costo mínimo. [4]

Estos modelos de redes pueden alcanzar una eficiencia mayor si son realizados bajo un escenario dinámico, esta integración se puede hacer a través de una proyección de densidad, la cual permite evaluar el modelo en un escenario más real y de esta manera ser más preciso al momento de plantear la solución.

Proyección poblacional: Las proyecciones de población constituyen una base fundamental para fines de planificación de las actividades económicas y sociales de un país. Permiten establecer los perfiles de las condiciones demográficas que llegaría a alcanzar una población, y por ende, evaluar las implicaciones que las nuevas situaciones van imponiendo.

Las proyecciones de población son, por lo tanto, el principal y más valioso instrumento para establecer anticipadamente la capacidad productiva y las necesidades básicas de una nación.

Los métodos de proyección se clasifican según la extensión de tiempo que han de cubrir sus resultados:

A. Proyecciones de corto plazo: No más de 5 años, son útiles en la elaboración de planes quinquenales.

B. Proyecciones de mediano plazo: Para períodos entre 10 y 15 años, son útiles en la formulación de proyectos globales de desarrollo.

C. Proyecciones de largo plazo: Para períodos de 20 años y más. Útiles para la formulación de análisis e interpretación de medidas o políticas demográficas.

Métodos para la elaboración de las proyecciones de población [5]:

A. Métodos matemáticos: Aplicación de modelos relacionales de cambio aplicable a problemas de población, tales como: función lineal, función geométrica, exponencial, logística, mínimos cuadrados, etc. Se considera que la población se comportará como una función matemática. Así mismo la interpolación y el ajuste de datos.

B. Métodos demográficos: Modelos que incluyen el análisis de los componentes del cambio demográfico: fecundidad, mortalidad y migraciones, se denomina "método de los componentes" y se 
apoya en la "Ecuación Compensadora" contemplándose el cambio pasado y reciente de estas variables, se realizan hipótesis de su comportamiento futuro. Utilizan como mínimo el sexo y la edad de la población, como variables básicas.

C. Métodos económicos: Se apoyan en las interrelaciones entre los aspectos (variables) socioeconómicos y las variables demográficas. Se utilizan para proyecciones geográficas. En este caso la formulación de hipótesis sobre el comportamiento demográfico, se apoya en el análisis de las variaciones socioeconómicas. Son menos usadas porque requieren de información socio-demográfica de partida, que no siempre se tiene disponible.

En cuanto a la prestación de servicios públicos existen leyes establecidas para determinar el costo de ese servicio.

En Colombia el modelo que se utiliza se rige a partir de la ley 142 de 1994[6] de la comisión de regulación de agua potable y saneamiento básico (CRA) a través del ministerio de medio ambiente. Esta ley es conocida como ley de servicios públicos domiciliarios.

En este modelo se tienen en cuenta las siguientes variables:

CMA: Son los gastos en que se incurre para administrar la entidad de manera que se pueda garantizar un servicio permanente a todos los usuarios.

CMO: Los costos de operación son aquellos necesarios para operar y mantener los sistemas de acueducto y alcantarillado, de manera que se pueda garantizar la prestación permanente del servicio a todos los usuarios.

CMI: El costo de inversión es aquel en que incurre una entidad prestadora del servicio para ampliar la capacidad de producción y suministro del sistema de acueducto y alcantarillado.

CMTAL: Constituye el costo por tasas ambientales, representado en las cargas contaminantes que son arrojadas a los vertimientos naturales.

Otro elemento a tener en cuenta dentro del modelo es la aplicación de subsidio para los estratos 1, 2 y 3 en el cual se plantea que se debe subsidiar el $70 \%$ al estrato $1,40 \%$ al estrato 2, y $6 \%$ al estrato 3 ; si el municipio no dispone de los recursos necesarios para subsidiar el servicio puede variar dichos porcentajes.

\section{DESCRIPCIÓN DEL PROBLEMA}

El caudal de aguas residuales de la ciudad objeto de estudio equivale aproximadamente al $80 \%$ del agua que se consume, es decir, $440 \mathrm{l} / \mathrm{s}$ de aguas residuales sobre $550 \mathrm{l} / \mathrm{s}$ de agua potable, de esta producción de aguas residuales el $20 \%$ es vertido al rio Guadalajara y el $80 \%$ al rio cauca, como se presenta en las figuras 1 y 2 que muestran los índices de contaminación por materia orgánica (residuos de origen doméstico e industrial) del rio Cauca [7].

\section{ICOMO - Índice de Contaminación por Materia Organica RIO CAUCA - 2 Semestre 2008}

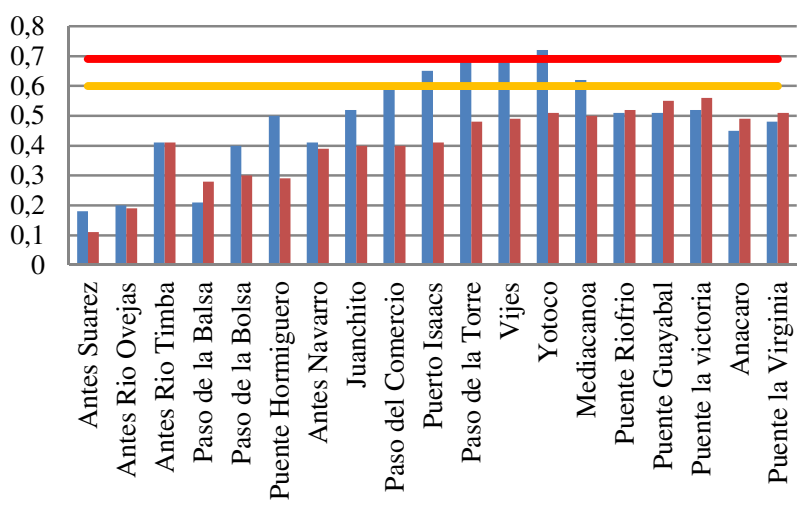

Fig. 1: Índices de contaminación orgánica. Fuente: CVC sep-nov 2008.

Como se puede ver en la gráfica los municipios más afectados por esta contaminación son Paso De La Torre, Vijes y Yotoco. El vertimiento de esta agua residual al río Cauca se hace a través de unas acequias que son alimentadas por colectores del sistema de alcantarillado de la ciudad estudiada. Este sistema de alcantarillado es uno de los más antiguos del departamento del Valle del Cauca, con colectores de más de 60 años en la zona colonial de tipo bóvedas en ladrillo, el cual ha venido ampliándose con planes de desarrollo previamente establecidos y por tanto, recibiendo cada vez más aguas residuales producidas por los asentamientos humanos [8].

El sistema de alcantarillado del casco urbano se encuentra sectorizado en dos áreas separadas por el río Guadalajara [9]. Como lo muestra la figura 2 : 
Tiene sus colectores dispuestos de oriente a occidente y de sur a norte.

\section{Colectores zona sur:}

A. Colector calle 1: Es el encargado de recoger las aguas residuales la zona sur de la ciudad (Albergue) y a él se une el colector Santa Rita, el cual, a su vez descarga las aguas residuales al colector de la calle 4.

El prestador del servicio de agua y alcantarillado es una empresa de servicios públicos mixta de acueducto y alcantarillado, la cual es la encargada de canalizar, potabilizar y distribuir el agua potable de la ciudad y recanalizar las aguas negras. Esta empresa es la encargada de prestar el servicio de agua y alcantarillado a 50 barrios [10] de la ciudad los cuales se dividen en 10 zonas como lo muestra la figura 2 y se puede observar porcentualmente en la figura 3 :

Fig. 2: Mapa de alcantarillado y zonas del municipio. Fuente: alcaldía municipal.

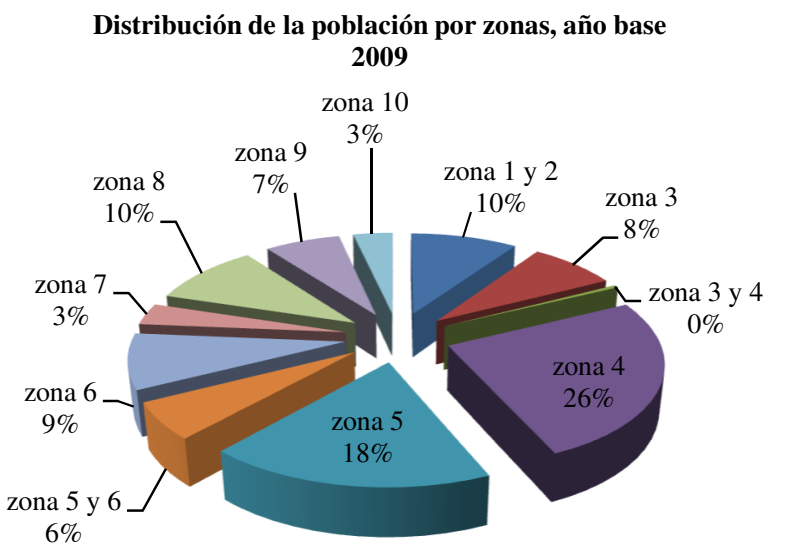

A. Colector calle 4: Este colector es el encargado de recoger todas las aguas residuales entre la calle $1 \mathrm{y}$ calle 6 de la ciudad o zona 8 .

B. Colector calle 8: Este colector es el encargado de recoger todas las aguas residuales entre la calle $7 \mathrm{y}$ calle 9 de la ciudad o zona 6 .

C. Colector calle 15 TIACUANTE: Este colector es el encargado de recoger todas las aguas residuales entre la calle 10 y calle 16 de la ciudad o zona 5 y tiene como destino final el río Cauca.

La ciudad también cuenta con dos colectores más, los cuales están ubicados en la calle 23 llamado colector pachita y otro ubicado en la calle 30 llamado colector honda, el colector honda se une al colector pachita y estos van directamente al rio Cauca. Estos colectores son de tipo mixto lo cual indica que recoge aguas lluvias y aguas residuales.

\section{Sector Sur}

Fig. 3: Porcentaje de distribución de la población por zonas año base 2009. Fuente: alcaldía municipal.

De igual manera el municipio se encuentra distribuido en estratos del 1 al 6, el mayor porcentaje de suscriptores se encuentran en estrato 2 (ver figura 4):

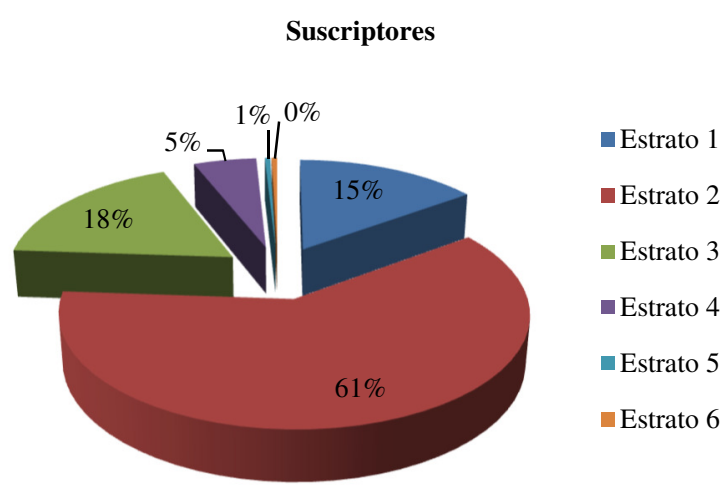


Fig. 4: Porcentaje de distribución de suscriptores por estrato. Fuente: Alcaldía municipal.

Actualmente la ciudad cuenta con estas tres propuestas para la localización de la PTAR ubicadas en diferentes partes de la ciudad las cuales son [8]:

\section{A. Alternativa 1}

Esta alternativa considera que la planta de tratamiento de aguas residuales PTAR, se localice en el occidente de la ciudad (barrio Paloblanco).

\section{B. Alternativa 2}

Corresponde básicamente a una modificación al trazado del emisor sanitario, desplazándolo más hacia el occidente, con el propósito de mejorar la pendiente del emisor y disminuir las excavaciones.

\section{Alternativa 3}

Implementación de dos PTAR para la ciudad de Buga, una en la zona norte y la otra en la zona sur que suplan las necesidades de cada zona.

\section{DISEÑO DE LA PROPUESTA}

Se realiza la construcción de un modelo de costos de transporte, tarifas y proyección poblacional para la evaluación de alternativas de localización de la PTAR.

\section{MODELO MATEMÁTICO}

El modelo matemático formulado en GAMS está constituido por los siguientes elementos:

- Conjuntos: Se definen por los elementos claves que forman el modelo y a partir de los cuales se declaran variables y ecuaciones en función de ellos. Para el presente trabajo, estos elementos claves son el conjunto de barrios lo cuales son los puntos entre los cuales se transporta el flujo y el conjunto PTAR que son los puntos definidos como destino final para el transporte del flujo.

Sean los índices: $\mathrm{i}=$ Barrios, $\mathrm{j}=$ PTAR

- Parámetros: Además de tener variables y relaciones entre las mismas, es decir ecuaciones, ha de alimentarse de ciertos valores numéricos constantes que denominaremos parámetros. Están asociados a los elementos de los conjuntos (SET) y sirven normalmente para indicar disponibilidades $\mathrm{O}$ requerimientos de estos.

$a_{i}=$ Capacidad de producción de los barrios i.

$b_{j}=$ Capacidad máxima de tratamiento de las PTAR $\mathrm{j}$.
$C_{i j}=$ Costo de transporte del barrio i a PTAR $\mathrm{j}$

- Datos: Es muy importante el manejo de los datos, se pueden manejar una TABLE la cual proporciona una forma fácil de introducir los datos del problema. Para el proyecto se manejará una TABLE donde se introducirán las tarifas de servicio.

- Variables de Decisión: Aquí, definiremos que variables vamos a emplear y de qué tipo es cada variable. Para el caso de estudio la variables de decisión será el flujo transportado de los barrios a las diferentes localizaciones de la PTAR ya que este viene asociado a un costo determinado por las normas tarifarias de alcantarillado actuales.

$x_{i j}=$ Flujo transportado entre barrios i y PTAR $\mathrm{j}$.

- Declaración de Ecuaciones: En esta parte se pueden definir las ecuaciones del modelo. Siempre es necesario definir un tipo especial de ecuación que es la función objetivo, que necesita de la participación de una variable especial que es la que hemos de minimizar en el proceso de optimización.

\section{- Función objetivo:}

La función objetivo está dada por:

$$
\sum_{i}^{m} \sum_{j}^{n} C_{i j} * x_{i j}
$$

Donde $i=1, \ldots, m ; j=1, \ldots, n$

El modelo propuesto plantea un costo tarifario que trae consigo tarifas reglamentadas por la ley que incluye costos de inversión, costos de tasas ambientales, de operación y administración.

\section{- Restricciones:}

i. Límite de capacidad máxima de producción para cada barrio i.

$$
\sum_{i}^{m} x_{i j} \geq a_{i}
$$

ii. Satisfacción de la demanda para cada localización j.

$$
\sum_{i}^{m} x_{i j} \leq b_{j}
$$

iii. Restricciones de no negatividad.

$$
x_{i j} \geq 0
$$




\section{IMPLEMENTACIÓN}

A partir de la información obtenida y explicada a través de la situación actual, se realizó el modelo de programación lineal desarrollado en el Sistema General de Modelización Algebraica "GAMS" por sus siglas en inglés, la herramienta utilizada para la solución del modelo es el método simplex.

Para dar cumplimiento al objetivo central del presente proyecto, se hizo necesario incorporar una estructura de orden lógico y sistemático, dividida en seis (6) fases, que constituyen la metodología empleada para la evaluación de las alternativas de localización.

Con la información del último censo de la totalidad de habitantes por barrio, se procedió al correspondiente análisis y proyección. Se proyectó mediante el promedio [12] de los tres modelos de referencia (aritmético, geométrico y Wappaus) ver figura 5:

\section{Año Base}

Promedio: (Aritmético, Geométrico, Wappaus)

POBLACIÓN PROYECTADA
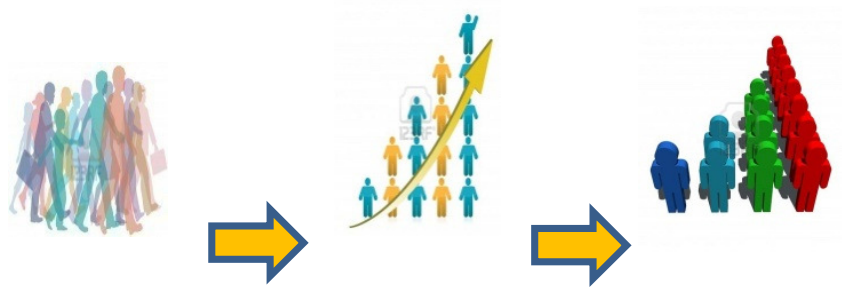

2009

2020

116.156 Hab

116.307 Hab

Fig. 5: Proyección poblacional promedio de los tres modelos referentes de proyección. Fuente: Elaboración propia.

\section{TARIFAS DE SERVICIO DE ALCANTARILLADO DE BUGA}
A. CMA: $\$ 2.475$ /suscriptor mes.
B. CMO: $\$ 526 / \mathrm{m}^{3}$ mes.
C. CMI: $\$ 47 / \mathrm{m}^{3}$.
D. CMTAL: $\$ 47 / \mathrm{m}^{3}$
E. CMLP: $\$ 1.844 / \mathrm{m}^{3}$

TARIFAS SUBSIDIADAS: De acuerdo a lo establecido por el gobierno municipal de la ciudad objeto de estudio, la empresa prestadora del servicio subsidia los estratos de acuerdo a la tabla 1:

\begin{tabular}{|c|c|}
\hline \multicolumn{2}{|c|}{ PORCENTUALIZACIÓN } \\
\hline Estrato 1 & $30 \%$ \\
\hline Estrato 2 & $19 \%$ \\
\hline Estrato 3 & $6 \%$ \\
\hline
\end{tabular}

Tabla 1. Subsidio por estrato. Fuente: Alcaldía municipal de Guadalajara de Buga.

Las tarifas subsidiadas son determinadas para el año base (2009); estas tarifas también son sujetas a una proyección teniendo como base la variación del índice de precios al consumidor (IPC) y son proyectadas hasta el año 2020 que es lapso de tiempo para el cual se establece el estudio. A continuación se muestra dicha proyección, (ver tabla 2):

\begin{tabular}{|c|c|c|c|c|c|}
\hline \multicolumn{6}{|c|}{ Proyección de Tarifas } \\
\hline Año & 2009 & 2012 & 2015 & 2018 & 2020 \\
\hline $\begin{array}{l}\text { Tarifa cargo fijo } \\
\text { estrato } 1\end{array}$ & $\$ 1.733$ & $\$ 1.850$ & $\$ 1.731$ & $\$ 1.475$ & $\$ 1.257$ \\
\hline $\begin{array}{l}\text { Tarifa consumo } \\
\text { básico estrato } 1\end{array}$ & $\$ 1.291$ & $\$ 1.378$ & $\$ 1.289$ & $\$ 1.099$ & $\$ 936$ \\
\hline $\begin{array}{l}\text { Tarifa Consumo } \\
\text { Complementario } \\
\text { estrato } 1\end{array}$ & $\$ 1.844$ & $\$ 1.969$ & $\$ 1.842$ & $\$ 1.570$ & $\$ 1.338$ \\
\hline $\begin{array}{l}\text { Tarifa cargo fijo } \\
\text { estrato } 2\end{array}$ & $\$ 2.005$ & $\$ 2.141$ & $\$ 2.003$ & $\$ 1.707$ & $\$ 1.455$ \\
\hline $\begin{array}{l}\text { Tarifa consumo } \\
\text { básico estrato } 2\end{array}$ & $\$ 1.493$ & $\$ 1.595$ & $\$ 1.492$ & $\$ 1.271$ & $\$ 1.084$ \\
\hline $\begin{array}{l}\text { Tarifa Consumo } \\
\text { Complementario } \\
\text { estrato } 2\end{array}$ & $\$ 1.844$ & $\$ 1.969$ & $\$ 1.842$ & $\$ 1.570$ & $\$ 1.338$ \\
\hline $\begin{array}{l}\text { Tarifa cargo fijo } \\
\text { estrato } 3\end{array}$ & $\$ 2.327$ & $\$ 2.485$ & $\$ 2.324$ & $\$ 1.981$ & $\$ 1.688$ \\
\hline $\begin{array}{l}\text { Tarifa consumo } \\
\text { básico estrato } 3\end{array}$ & $\$ 1.733$ & $\$ 1.851$ & $\$ 1.731$ & $\$ 1.475$ & $\$ 1.258$ \\
\hline $\begin{array}{l}\text { Tarifa Consumo } \\
\text { Complementario } \\
\text { estrato } 3\end{array}$ & $\$ 1.844$ & $\$ 1.969$ & $\$ 1.842$ & $\$ 1.570$ & $\$ 1.338$ \\
\hline
\end{tabular}

Tabla 2. Proyección de las tarifas de acuerdo a la variación del IPC. Fuente: Elaboración propia.

\section{RESULTADOS}


Para el proyecto se debe plantear y correr dos escenarios, los cuales son:

Escenario 1: Analizar como interactúa el modelo con tarifas homogéneas sin subsidios, para generar un balance entre los barrios y los costos de cada localización; ya que el modelo se basa en el costo mínimo de transporte con iteración de cada barrio para dar una solución óptima de localización.

Los resultados arrojados por el software GAMS nos indican que la solución factible es la alternativa de localización número 1, la cual presenta los valores medios (level) para los que la solución que se muestra es la más viable de las tres alternativas estudiadas; todo esto para el caso del análisis de la variable de decisión del flujo transportado $\left(x_{i j}\right)$ entre los barrios i y el destino PTAR j; ya que cumple con las restricciones de capacidad y satisfacción de la demanda propuestas en el desarrollo del modelo.

Los resultados arrojados por el programa indican que se ha encontrado una solución óptima con un valor de \$27’928.852.371 correspondiente a la variable de decisión de costo de transporte $(C T)$ y para ello se ha realizado 62 iteraciones de las $2000^{\prime} 000.000$ posibles.

Escenario 2: Analizar y correr el modelo con tarifas subsidiadas por el municipio caso de estudio, sabiendo que el $61 \%$ de los suscriptores pertenecen al estrato 2 , el $18 \%$ al estrato 3 y el $15 \%$ al estrato 1 , lo cual representa $94 \%$ de la población subsidiada, todo esto para ajustarlo a la realidad del municipio en la actualidad y apegándose a la ley de los servicios públicos como lo dice el artículo 142.

Los resultados arrojados por el software GAMS nos indican que la solución factible es la alternativa de localización número 1, la cual presenta los valores medios (level) para los que la solución que se muestra es la más viable de las tres alternativas estudiadas; todo esto para el caso del análisis de la variable de decisión del flujo transportado $\left(X_{i j}\right)$ entre los barrios i y el destino j PTAR; ya que cumple con las restricciones de capacidad y satisfacción de la demanda propuestas en el desarrollo del modelo bajo el escenario de los costos de transporte que se le aplica subsidio para los estratos uno (1), dos (2) y tres (3).

Los resultados arrojados por el programa indica que se ha encontrado una solución óptima con un valor de \$9871'293.894 y para ello se ha realizado 63 iteraciones de las 2000 '000.000 posibles.

Esta alternativa según lo contempla el plan estratégico que elaboró la firma COLOMBIANA DE INGENIERÍA Y ARQUITECTURA LIMITADA-CIA LTDA, considera se localice en el sitio previsto al occidente de la ciudad marcado con el círculo rojo como lo muestra la figura 6 :

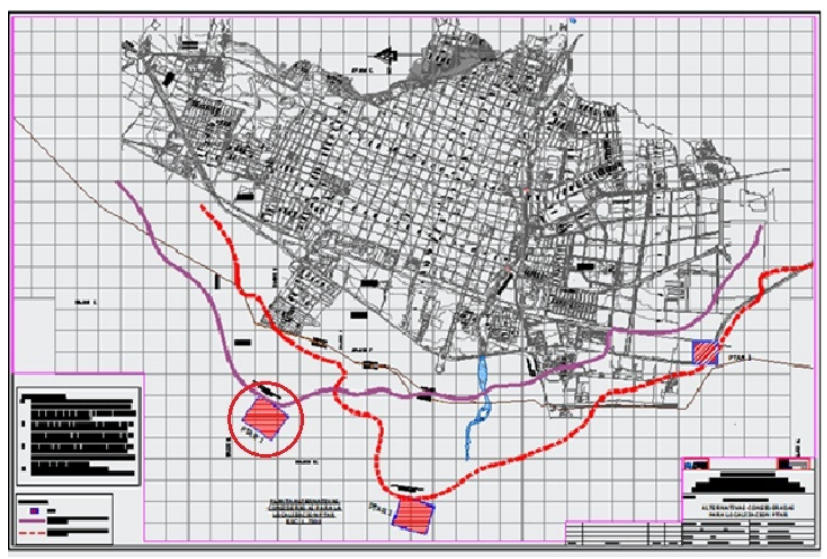

Fig. 6: Solución factible. Fuente: Alcaldía municipal

\section{v1. CONCLUSIONES}

El crecimiento de la población caso de estudio presenta un nivel que si bien no varía demasiado, si tiene incidencia en la generación de residuos sólidos que son arrojados directamente a los afluentes, como se demostró en el estudio realizado el cual muestra un aumento constante de estos residuos empezando en el año 2009 año base con una

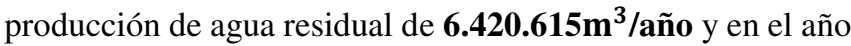
2020, año final de periodo de estudio con 7.163.277 $\mathbf{~ m}^{3} / \mathbf{a n ̃ o}$; presentando un aumento de $\mathbf{7 4 2 . 6 6 2} \mathbf{~ m}^{3} / \mathbf{a n ̃ o}$ de agua residual.

El modelo tarifario establecido para el cobro del servicio de alcantarillado, es el empleado por el gobierno nacional, el cual está reglamentado y plantea el tipo de cobro a realizar y como debe hacerse, para la aplicación de la tarifa se tienen en cuenta factores como el número de habitantes, la aplicación de subsidios entre otros; cabe anotar que el cobro del servicio de alcantarillado se aplica como un porcentaje del cobro del servicio de acueducto, el cual es del $30 \%$ para el caso del problema planteado.

El modelo empleado para dar solución al problema de localización cumple con su fin, ya que logra determinar los costos de transportar el flujo hasta cada una de las posibles localizaciones y arroja como resultado, cuál de estas posibles localizaciones presenta el menor costo de esta manera permite definir la solución más factible de localización de la planta de tratamiento de aguas residuales en la ciudad caso de estudio.

Se debe tener en cuenta que al momento de la construcción de la PTAR, se debe definir la capacidad de flujo que va a soportar está planta, ya que este flujo irá cambiando a través del tiempo y se verá afectado directamente por la población, 
por este motivo para el municipio es de mayor importancia que la PTAR sea construida con base a la población futura, no a la actual; ya que en pocos años la planta de tratamiento no podría tratar toda el agua residual del municipio.

Más allá de lo explicado matemáticamente en este proyecto, se debe tener conciencia que la localización de la planta de tratamiento no puede dar más espera, el índice de contaminación actual de los afluentes está creciendo de manera alarmante, contaminando de forma irreversible el agua.

\section{REFERENCIAS}

[1] N.P. Loomba, Linear Programming: An introductory analysis, New York, McGraw-Hill, 1964.

[2] A. Klose y A. Drexl, Facility location models for distribution system design, Switzerland, 2004.

[3] M.F. Goic, Flujo de redes, Clase Auxiliar, Universidad de Chile.

[4] F.S. Hillier y G.J. Lieberman, Investigación de operaciones, Séptima edición, McGraw-Hill, 2001
[5] M. Rincón, Teoría y Métodos para la Preparación de Estimaciones y Proyecciones de Población, 2000.

[6] Ministerio de Ambiente, Vivienda y Desarrollo Territorial, Costos y tarifas. Municipios menores y zonas rurales, 2005.

[7] Corporación Autónoma Regional del Valle del Cauca. "Principales corrientes superficiales del valle del cauca, índices de calidad del agua",

[8] Colombiana de Ingeniería y Arquitectura Limitada- CIA. LTDA., "Estudio y diseños para el plan maestro de acueducto y alcantarillado de la zona sur y norte de la ciudad de Buga con proyección al año 2017”.

[9] Gandini y Orozco LTDA., Corporación autónoma regional del Valle del Cauca, Plan maestro de alcantarillado.

[10] Diagnostico para el plan de desarrollo municipal. Municipio de Buga, 2004-2007.

[11] Informe de diagnóstico y factibilidad. Municipio de Buga. Contrato de consultoría No. 011. 2006. 